\title{
Early Recurrence Improves Edge Detection
}

Xun Shi

shixun@cse.yorku.ca

Bo Wang

wangbo.yunze@gmail.com

John K. Tsotsos

tsotsos@cse.yorku.ca

\author{
Department of Computer Science and \\ Centre for Vision Research (CVR) \\ York University \\ Toronto, Ontario, Canada
}

\begin{abstract}
A biologically motivated computational model of early recurrence is proposed for edge detection. Studies of the primate vision suggested that visual features are transmitted in the two visual pathways with different speeds (with the dorsal pathway processing faster than that of the ventral pathway) and the presences of extensive recurrent connections across the two pathways. It is thus likely that the dorsal perception facilitates the ventral perception via early recurrent mechanism. Following these neural principles, we hypothesize that early recurrence enables responses to high-spatial frequency features (fine edges) to be suppressed by low-spatial frequency features (coarse edges) in a multiplicative manner. Using real images, we quantitatively compared contours calculated by our work with another well-known biologically motivated model. To further explore early recurrence in solving machine vision problems, the representation is used to boost different popular edge algorithms. Results from both experiments lead to the conclusion that early recurrence has a positive and consistent influence on edge detection.
\end{abstract}

\section{Introduction}

Edge detection is an essential task in image analysis and machine vision. A number of

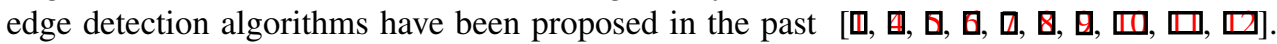
However, the problem remains partially resolved. Perhaps the most difficult challenge is how to generate an edge map to accurately localize true edges that are perceptually salient, and meanwhile to exclude false edges caused by image noises and textures.

This paper concerns a computational model of early recurrence for edge detection. The model is motivated by studies of primate vision. It is proposed that visual features are perceived through the two main visual pathways, namely the dorsal and the ventral pathway, with different speeds: the dorsal pathway processes information much more rapidly than that of the ventral pathway. Depending on their physiological properties [ $\square]$ ], the dorsal pathway is sensitive to low-spatial frequency features (e.g. coarse edges) and the ventral pathway is sensitive to high-spatial frequency features (e.g. fine edges). In traditional views, edges are perceived only in the ventral pathway, particularly in the ventral layers of the primary visual cortex (V1), or ventral V1. However, recent studies of asynchronous cortical processing have suggested that computation in ventral V1 may be influenced by signals coming from higher-level dorsal areas as a result of massive early recurrent mechanisms [ष, $\square, \square, \square]$. 


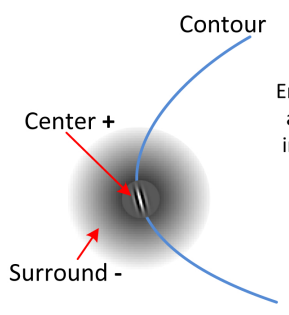

Center-surround self-inhibition

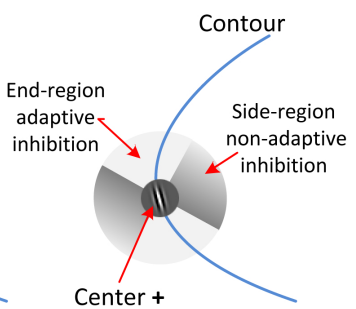

Butterfly-shaped self-inhibition

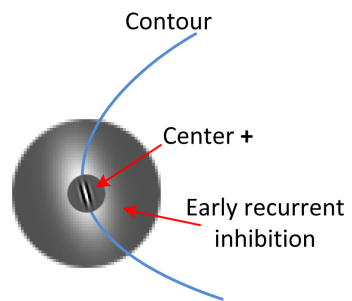

Proposed early recurrent inhibition

Figure 1: Compare non-classical RF inhibition mechanisms. Left: self-inhibition [四], middle: butterfly-shaped self-inhibition [ष]], right: the proposed early recurrent inhibition.

We formalize a computational model to the aforementioned early recurrent mechanism. The computation simulates the early recurrence from dorsal area MT to ventral V1. The essence of the mechanism is that responses of ventral V1 are inhibited by responses of MT. We hypothesize that the inhibition facilitates the low-level feature computation and leads to a much cleaned edge representation. ${ }^{1}$ It is proposed that early recurrent inhibition is a weighted multiplication operation. Depending on the weighting strategy, two instances of MT-ventral V1 recurrence are investigated, namely isotropic and anisotropic inhibition. Isotropic inhibition causes ventral V1 responses to a preferred orientation to be inhibited by the sum of MT responses to all orientations in an equal manner. Anisotropic inhibition suppresses ventral V1 responses to a preferred orientation by MT responses to the same orientation. Our proposed model is consistent with the scale-space theory [पष] and suggests that cross-pathway interaction plays a role in enforcing consistency of image structure across scales. The model includes an interval-tree based representation, which highlights a marked correspondence between the stability of object contour and its perceptual salience.

To investigate the performance in practical machine vision applications, refined edge representation was used as input to a contour operator proposed in [四]. Using real images, we quantitatively compared contours calculated by our work with the biologically motivated contour detector in [四]. In addition, we applied early recurrent presentation to boost different popular edge representations. Results from both experiments clearly demonstrate that early recurrent inhibition has a positive and consistent impact on the contour detection.

The rest of the paper is organized as the follows. Section 2 reviews related work. Section 3 proposes and formalizes the model of early recurrence. Section 4 discusses experiment and results. Section 5 concludes the work.

\section{Related Work}

Existing edge models compute edges by first-order [ $[$,, Q] $]$ or second-order approaches [ $[$ ] to search for pixel value changes. To discriminate true edges associated with primitives from false edges due to image textures and noises, approaches of statistical analysis [ $\square$, 罒, multi-

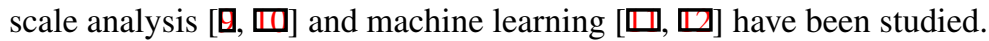

Inspired by the primate visual system, a number of models have been proposed that make use of neuronal non-classical RF characteristics to facilitate edge detection. In [四,

\footnotetext{
${ }^{1}$ Although literature commonly models the dorsal pathway as sensitive to spatiotemporal features, numerous neurophysiological studies $[\square, \square, \square]$ have confirmed that dorsal area MT also responds to static image features.
} 
the center-surround mechanism found in many V1 neurons is modeled as self-inhibition that suppresses edge segments observed surround the center RF (see Fig. 1 left drawing). The authors formalized two types of self-inhibition, isotropic and anisotropic. Experiments using real images confirmed that self-inhibition improves edge detection, leading to much cleaned contour maps compared with Canny and Gabor energy edge detectors. Investigation of selfinhibition has been extended into multi-scale analysis in $[\square, \mathbf{Q}]$ with different applications. More recently self-inhibition has been re-visited with a refined center-surround inhibition scheme [ष]. A butterfly-shaped surround region is proposed, which consists of two adaptive inhibitory end-regions and two non-adaptive inhibitory side-regions (see Fig. 1 middle). Experiments showed that the surround region performs better than [四] in preserving real image contours. However, the biological underpinnings to such adaptive self-inhibition region are unclear. Although the authors have discussed a few possible sources of inhibition, they did not explain which visual areas or cortical connections the model represents.

\section{Computational Model of Early Recurrence}

\subsection{General Model}

The cross-pathway recurrent mechanism observed in our brain inspires us with an alternative kind of inhibition that may be useful in improving edge representation. Our model views the primate visual system as a multi-layered two-visual-pathway representation [四, 四]. The two visual pathways compute different edge features. Specifically, ventral V1 computes fine-scale edges and projects results to higher-level ventral areas to compute object contour. Dorsal V1 (the dorsal layers of area V1) is sensitive to coarse-scale edges and sends output to area MT for further analysis. Results of MT are fed back to modulate ventral V1 computation (Fig. 2 left drawing). Modulated ventral V1 responses are further inhibited by the centersurround self-inhibition [四]. The whole process can be defined as:

$$
R^{V 1 v}=H\left(E^{V 1 v} \cdot \operatorname{Inh} h_{r e}^{M T}-\alpha \operatorname{Inh} h_{c s}^{V 1 v}\right),
$$

where $R^{V 1 v}$ denotes ventral V1 representation. $H(s)=\max (s, 0)$ is a half-wave rectification function. $E^{V 1 v}$ denotes ventral V1 responses to feed-forward image stimuli, which have been commonly deemed as the edge representation in most existing works. $I n h_{r e}^{M T}$ is the early recurrent inhibition generated from area MT. $I n h_{c s}^{V 1 v}$ denotes the center-surround selfinhibition, and $\alpha$ is a weighting factor.

Experiments $[\square, \mathbb{Z}$, have suggested that the biophysical underpinnings of MT-V1 recurrence can be described by multiplication. We thus propose $I n h_{r e}^{M T}$ inhibits V1 via multiplication. Further, $\operatorname{Inh} h_{c s}^{V 1 v}$ is defined as isotropic Non-Classical Receptive Field inhibition

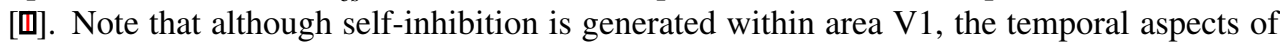
the asynchronous signal projection properties between the dorsal and the ventral pathways make it possible that the recurrent inhibition from MT impacts ventral V1 at an earlier time. Therefore, recurrent inhibition performs prior to self-inhibition (Fig. 2).

\subsection{Formalization}

\subsubsection{V1 response to feed-forward visual stimuli}

V1 neurons is modeled as a bank of 2D Gabor filters. Each filter is constructed as:

$$
g_{\lambda, \sigma, \psi}^{V 1}(x, y, \theta)=e^{-\left(\tilde{x}^{2}+r^{2} \tilde{y}^{2}\right) /\left(2 \sigma^{2}\right)} \cos (2 \pi \tilde{x} / \lambda+\psi),
$$




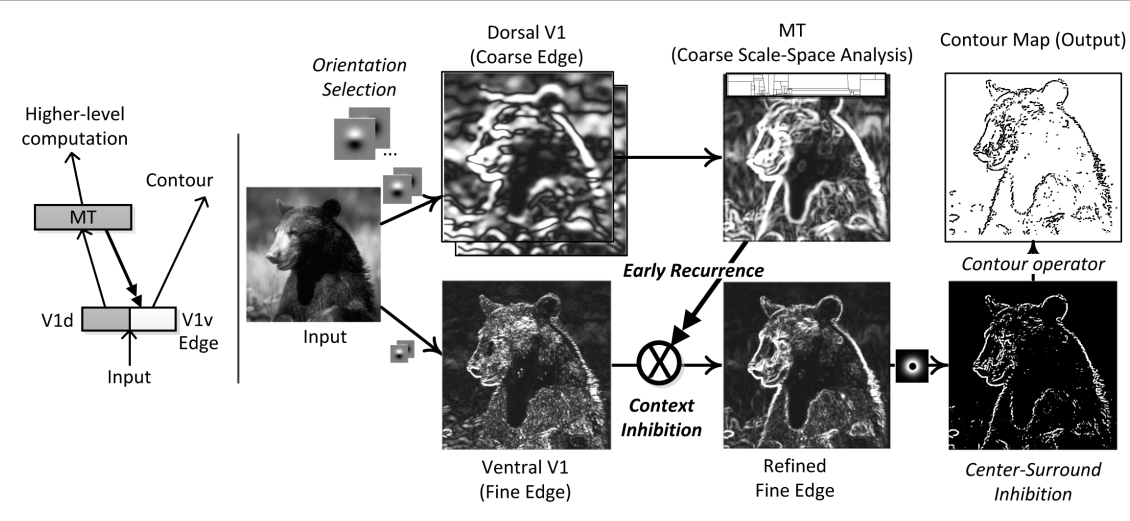

Figure 2: General structure of the proposed model. Left: simplified biological hierarchy and connections. The double arrow line from MT to V1v denotes the early recurrence. Right: an example of using the proposed computational model to compute object contours.

where, $\tilde{x}=x \cos \theta+y \sin \theta, \tilde{y}=-x \sin \theta+y \cos \theta$, and $\theta$ is the preferred orientation. In our study $N=12$. $\psi$ is the phase offset. $r$ is the aspect ratio of the eccentricity of the Gaussian. According to [ $\square]$, we set $r=0.5$. $\sigma$ denotes the standard deviation of the Gaussian. $\lambda$ is the wavelength. According to $[\mathbb{\nabla}]$, most V1 neurons have a bandwidth between 1.0 and 1.5 octaves, therefore $\sigma / \lambda=0.56$ is used.

Given an input image $I(x, y)$, V1 response is formalized as:

$$
r_{\lambda, \sigma, \psi}^{V 1}(x, y, \theta)=I(x, y) * g_{\lambda, \sigma, \psi}^{V 1}(x, y, \theta),
$$

where $*$ denotes convolution. To deal with phase shift, the quadrature pair technique is used, which integrates results of Gabor filtering as:

$$
E_{\lambda, \sigma, \psi}^{V 1}(x, y, \theta)=\sqrt{\left(r_{\lambda, \sigma, \psi}^{V 1}\right)^{2}+\left(r_{\lambda, \sigma, \psi+\pi / 2}^{V 1}\right)^{2}},
$$

In our experiment, $\sigma$ values corresponding to dorsal $\mathrm{V} 1\left(\sigma_{c}\right)$ and ventral $\mathrm{V} 1\left(\sigma_{f}\right)$ are set differently to characterize preferred spatial sensitivities (scale of edge).

\subsubsection{MT integration of dorsal $\mathrm{V} 1$ activation}

Area MT integrates feed-forward activations from dorsal V1 and sends results to inhibit ventral V1. In its simplest manner, area MT is modeled using a Gaussian:

$$
g_{\sigma_{M T}}^{M T}(x, y)=e^{-\left(x^{2}+r^{2} y^{2}\right) /\left(2 \sigma_{M T}^{2}\right)},
$$

where $\sigma_{M T}$ denotes the standard deviation of the Gaussian. Output of MT is therefore:

$$
r_{\lambda, \sigma, \psi}^{M T}(x, y, \theta)=E_{\lambda, \sigma, \psi}^{V 1}(x, y, \theta) * g_{\sigma_{M T}}^{M T}(x, y),
$$

\subsubsection{Scale-Space Representation}

To search for best coarse scales, we notice that the output of Eq. (4) is a scale-space representation $(x-y-\theta)$. We extended single-scale MT representation to formalize an algorithm that employs the interval-tree analysis [ $\square]$ to determine the coarse-level edge representation. 


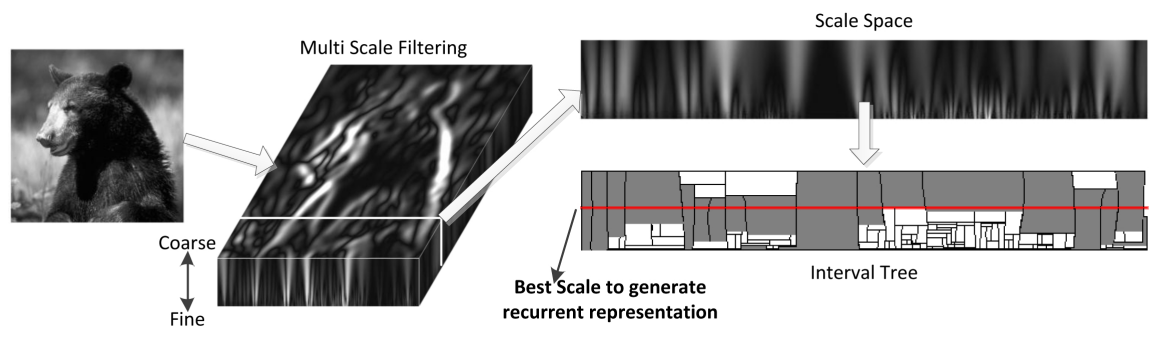

Figure 3: Scale-Space Analysis. Given an input image, the scale-space representation is computed coarse-to-fine. To simplify the work, the three dimensional scale-space $x-y-\theta$ is separated into $x-\theta$ and $y-\theta$ respectively. An interval tree is built following [ष]], based on which the best coarse-scale representation is determined via searching for a covering of the space (gray blocks).

To simplify the work, the 3-D scale-space is separated into 2-D scenarios, $x-\theta$ and $y-\theta$. To further reduce the computational costs, only two gradient of Gaussian $\left(0^{\circ}\right.$ and $\left.90^{\circ}\right)$ are used. They are defined as:

$$
\begin{aligned}
& \nabla_{x} g^{M T}\left(x, y, \sigma_{g x}\right)=\left(-x / \pi \sigma_{g x}^{2}\right) e^{-\left(x^{2}+r^{2} y^{2}\right) /\left(2 \sigma_{g x}^{2}\right)} \\
& \nabla_{y} g^{M T}\left(x, y, \sigma_{g y}\right)=\left(-y / \pi \sigma_{g y}^{2}\right) e^{-\left(x^{2}+r^{2} y^{2}\right) /\left(2 \sigma_{g y}^{2}\right)}
\end{aligned}
$$

By increasing $\sigma_{g x}$ and $\sigma_{g y}$, scale-space representations $x-\theta$ and $y-\theta$ can be constructed respectively. As shown in Fig. 3, given a $x-\theta$ representation, an interval-tree is built following [ष]. It is observed that scales of real object contours have a marked correspondence with the stability (vertical axis) in the scale-space. Therefore, the algorithm searches for a covering of the space (a set of gray blocks) that includes the most stabled blocks across all interval (horizontal axis). The algorithm then selects the largest scale $\hat{\sigma_{g x}}$ that cross the most blocks in the covering as the output scale, and output of MT of $0^{\circ}$ is formalized as:

$$
r^{M T}(x, y, 0)=\nabla_{x} g^{M T}\left(x, y, \hat{\sigma_{g x}}\right),
$$

The same operation also applies on $y-\theta$ space.

\subsubsection{Early recurrent inhibition $I n h_{r e}^{M T}$}

We propose that early recurrent inhibition between MT and ventral V1 can be represented as a weighted multiplicative process. The inhibition representation is defined as:

$$
\operatorname{Inh} h_{r e}^{M T}(x, y, \theta)=\frac{\sum_{\delta \in \Delta} \omega_{(\delta, \theta)} r_{\lambda, \sigma, \psi}^{M T}(x, y, \theta)}{\left\|\sum_{\delta \in \Delta} \omega_{(\delta, \theta)} r_{\lambda, \sigma, \psi}^{M T}(x, y, \theta)\right\|_{1}},
$$

where $\omega_{(\delta, \theta)}$ is the weighting factor, denoting strength of connection between MT neuron of orientation $\delta$ and ventral V1 neuron of orientation $\theta$. $\sum$ denotes the summation of MT to all orientations $\delta \in \Delta$. \|\|$_{1}$ is the L1 norm. By setting $\omega_{(\delta, \theta)}$, two special types of early recurrent inhibition scheme are derived and investigated separately.

R1. Isotropic inhibition causes fine-scale edge of an orientation inhibited by MT with all orientations in an equal manner. To do this, we fix $\omega_{(\delta, \theta)}=1$ for all orientations. The isotropic representation is a summation of MT. It highlights regions corresponding to lowspatial frequency variations to all orientations and is insensitive to variations caused by highfrequency stimuli (i.e. noise and textures) . 
R2. Anisotropic inhibition suppresses fine-scale edge to a preferred orientation by MT responses to the same orientation. This is done by setting:

$$
\omega_{(\delta, \theta)}= \begin{cases}1 & \text { if } \delta=\theta \\ 0 & \text { otherwise }\end{cases}
$$

Each MT representation contains information of low-spatial frequency variations to only one orientation. It then modulates V1 responses to the same orientation.

\subsubsection{Center-Surround Isotropic Self-inhibition}

Center-surround isotropic self-inhibition, $\operatorname{Inh} h_{c s}^{V 1 v}$ is formalized following [四] as a convolution of the maximum energy map $\hat{E}_{\lambda, \sigma, \psi}^{V 1}(x, y, \theta)$ with a weighting function as:

$$
\operatorname{Inh}_{c s}^{V 1 v}=\hat{E}_{\lambda, \sigma, \psi}^{V 1 v}(x, y) * w_{\sigma}^{V 1 v}(x, y),
$$

where the maximum energy map is calculated as:

$$
\hat{E}_{\lambda, \sigma, \psi}^{V 1 v}(x, y)=\max \left\{E_{\lambda, \sigma, \psi}^{V 1 v}\left(x, y, \theta_{i}\right) \mid i=1 . . N\right\}
$$

and the weighting function is defined with Difference of Gaussian function [ $[\mathrm{Q}]$ as:

$$
w_{\sigma}^{V 1 v}(x, y)=\frac{H(\operatorname{DoG}(x, y))}{\|H(\operatorname{DoG}(x, y))\|_{1}},
$$

\section{Experiment}

The proposed model has been implemented into three forms: (fI) isotropic inhibition based on single-scale recurrence, (fA) anisotropic inhibition based on single-scale recurrence, and (ssI) isotropic inhibition based on scale-space recurrence ${ }^{2}$. A contour operator is implemented [四] to produce binary contours.

A dataset of 40 images with hand-drawn ground-truth contours are used for performance evaluation $^{3}$. This dataset has been widely used in the literature of contour detection. Although there are other datasets for edge detection, images in this dataset cover a broad range of spatial frequency variations, different types of textures and image noise.

To investigate the effect of early recurrence, we design the first experiment that compares our model with another well-known biologically inspired edge detector [四]. Their method of isotropic center-surround self-inhibition (S) has been implemented. Detected contours are compared against ground-truth contours. The performance measurement introduced in [四] is employed with False Positive Rate (FP), False Negative Rate (FN), and Performance (P).

Each edge detector is tested with a total of 100 different combinations of parameters to investigate the relationships between different parameter settings. For $\mathbf{S}$ and $\mathbf{s s I}, 10 \sigma_{f}$ values are used in Eq. 2 from 0.5 to 3.2, with 0.3 interval, to generate fine-scale edge maps. For fI and $\mathbf{f A}, 5 \sigma_{f}$ values are used from from 0.5 to 3.2 , with 0.6 interval, and single coarse-scale $\sigma_{c}$ is defined as 4 and 6 times to the fine-scale $\sigma_{f}$. For all detectors, $10 \alpha$ values are used in Eq. 1 from 0 to 2.7 , with 0.3 interval, to investigate self-inhibition.

Fig. 4 compares the best contours of four selected images. These images contain objects of different types. From the comparisons of contour continuity, edge detail and background

\footnotetext{
${ }^{2}$ The other case, anisotropic inhibition, is not implemented since mismatched number of orientations. Note that we simplify in scale-space to use gradient of Gaussian with 2 orientations to compute the coarse-scale edge representation, but fine-scale edge maps are generated using Gabor filters with 12 orientations

${ }^{3}$ [Online Available] http://www.cs.rug.nl/ imaging
} 
Table 1: Experiment parameters and performance for the images presented in Fig. 4. Last row highlights P score improvements over $\mathbf{S}$ [四].

\begin{tabular}{|l|cccc|cccc|cccc|cccc|}
\hline \hline & \multicolumn{4}{|c|}{ Bear } & \multicolumn{4}{|c|}{ Elephant_2 } & \multicolumn{4}{c|}{ Rnu } \\
& S & fI & fA & ssI & S & fI & fA & ssI & S & fI & fA & ssI & S & fI & fA & ssI \\
\hline$\sigma_{f}{ }^{*}$ & 2.8 & 2.8 & 2.8 & 3.2 & 2.8 & 2.0 & 2.8 & 3.2 & 2.8 & 2.8 & 2.8 & 3.2 & 2.6 & 2.8 & 2.8 & 3.2 \\
$\sigma_{c}{ }^{*}$ & - & $6 \sigma_{f}$ & $6 \sigma_{f}$ & - & - & $6 \sigma_{f}$ & $6 \sigma_{f}$ & - & - & $4 \sigma_{f}$ & $4 \sigma_{f}$ & - & - & $6 \sigma_{f}$ & $4 \sigma_{f}$ & - \\
$\alpha$ & 0.9 & 0.6 & 1.2 & 0.6 & 0.9 & 1.2 & 0.0 & 0.0 & 0.9 & 0.0 & 0.3 & 0.0 & 1.2 & 0.9 & 1.2 & 0.0 \\
$F P$ & 2.51 & 1.52 & 0.67 & 0.52 & 0.61 & 0.24 & 0.48 & 0.28 & 0.65 & 0.31 & 0.32 & 0.13 & 0.36 & 0.68 & 0.33 & 0.49 \\
$F N$ & 0.56 & 0.33 & 0.43 & 0.20 & 0.36 & 0.43 & 0.32 & 0.35 & 0.53 & 0.46 & 0.43 & 0.35 & 0.44 & 0.30 & 0.40 & 0.18 \\
$P$ & 0.21 & 0.33 & 0.41 & 0.56 & 0.46 & 0.50 & 0.52 & 0.55 & 0.36 & 0.47 & 0.48 & 0.60 & 0.47 & 0.47 & 0.50 & 0.58 \\
Improve & - & $\mathbf{5 7 \%}$ & $\mathbf{9 5 \%}$ & $\mathbf{1 6 7 \%}$ & - & $\mathbf{9 \%}$ & $\mathbf{1 3 \%}$ & $\mathbf{2 0 \%}$ & - & $\mathbf{3 1 \%}$ & $\mathbf{3 3 \%}$ & $\mathbf{6 7 \%}$ & - & $\mathbf{0 \%}$ & $\mathbf{6 \%}$ & $\mathbf{2 3 \%}$ \\
\hline
\end{tabular}

${ }^{*} \sigma_{f}$ and $\sigma_{c}$ represent standard deviation $(\sigma)$ used in Eq. 2.

texture inhibition, it is clear that the contour maps computed by the proposed detectors achieve better performance than method from [四]. Table 1 lists parameters used and performance. Our proposed methods surpass the competitor in most performance measurements. The enhancements over self-inhibition detector are significant (as much as 167\%). The proposed isotropic inhibition achieves the lowest false positive rate, indicating a better suppression of false edges. The proposed anisotropic inhibition has the lowest false negative rate, which further confirms it has superior ability to retain real object contours.

Fig. 5 right compares $\mathrm{P}$ scores. The best performance (top bar) and median performance (red line in box) of the proposed detectors are consistently higher than $\mathbf{S}$. Due to single coarse-scale $\sigma_{c}$, early recurrent inhibition of $\mathbf{f A}$ and $\mathbf{f I}$ are not as stable as ssI.

To further investigate whether the proposed model is a general process for machine vision, we incorporate the scale-space analysis based recurrent representation into 2 popular edge detectors. In particular, Canny edge detector [ $⿴ 囗 十$ is chosen because it is widely used edge detector, and multi-scale Brightness/Texture Gradients (BTG) detector proposed in [D] is selected because it represents a state-of-the-art approach. Although Malik and his colleagues have extended the work [ $\square, \square]$, the goals of these extensions emphasize learning to detect contours, and thus they do not confine to the current purpose. The proposed recurrent representation fits itself into these models by modulating original edge responses before constructing contour, which produced modulated detector Canny+ER and BTG+ER.

We tested these methods on the same dataset (Fig. 6), which indicated that via modulation, edges detected more focus on real contours. ROC and Precision-Recall curves generated based on ground truth show that modulated edges augment baselines in most cases. The proposed computational model may consistently improve edge detections in real scenes.

\section{Conclusion}

In this paper, we proposed a computational model of early recurrence to improve edge detection. The model is inspired by the asynchronous visual feature processing mechanism found in the primate visual system. The computation uses dorsal (coarse) edge to inhibit ventral (fine) edges by multiplicative inhibition. The proposed recurrent inhibition is fundamentally different from Self-inhibition [四], which is a mechanism within area V1 and works in an additive manner. Early recurrence, alternatively, uses an additional kind of inhibition generated from dorsal area MT and works by multiplication.

The first experiment examined soundness of early recurrence based on a filter parameter study. The second experiment applied early recurrent to boost practical contour detectors. Using real images, results indicate that contours generated via early recurrence consistently surpass those in [四] and machine vision algorithms [ $[\square, \square]$. Isotropic and anisotropic inhi- 


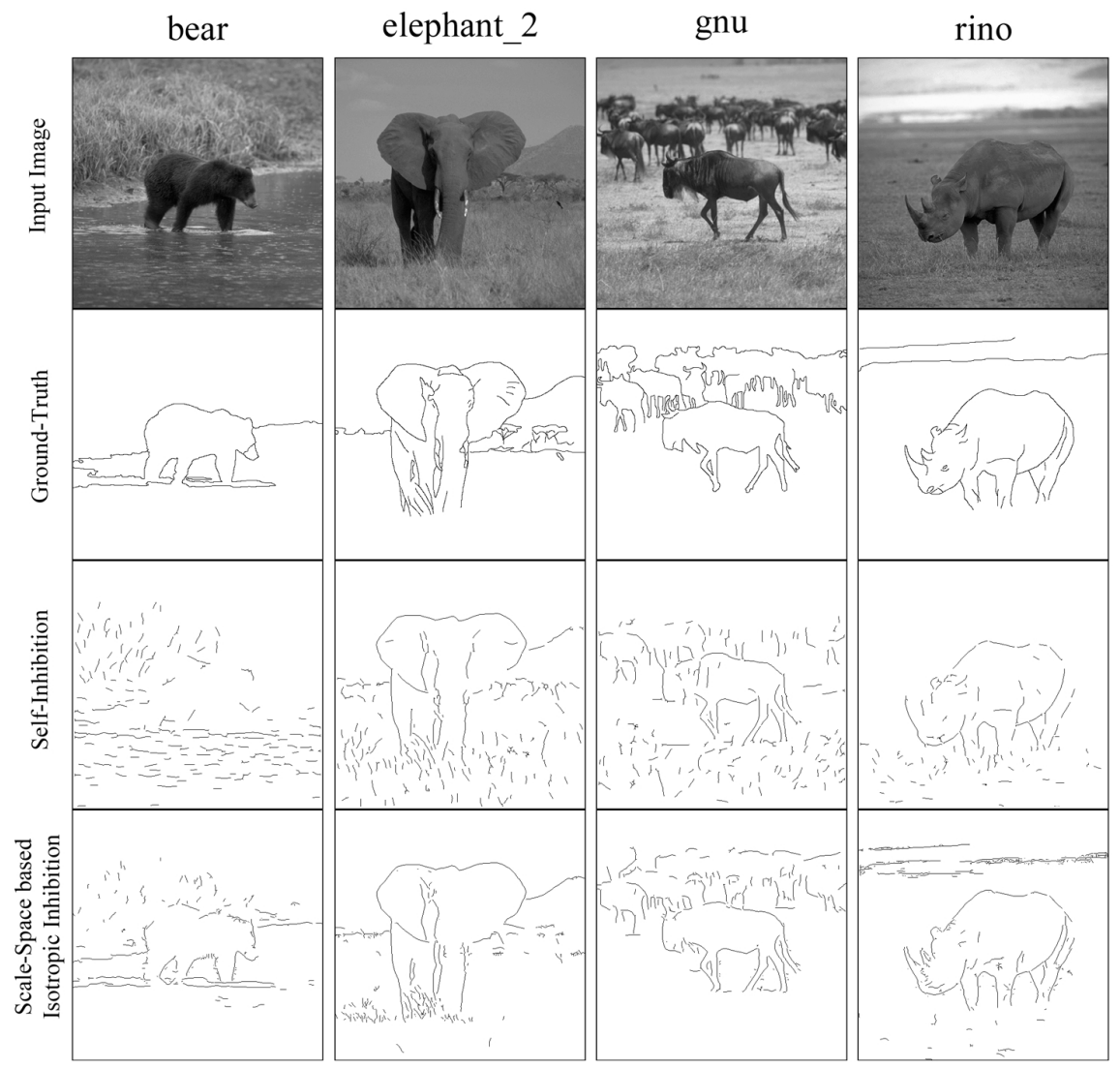

Figure 4: Contours comparison from top to bottom, input image, ground truth, computed contours by $\mathbf{S}$ [四 and scale-space analysis ssI.
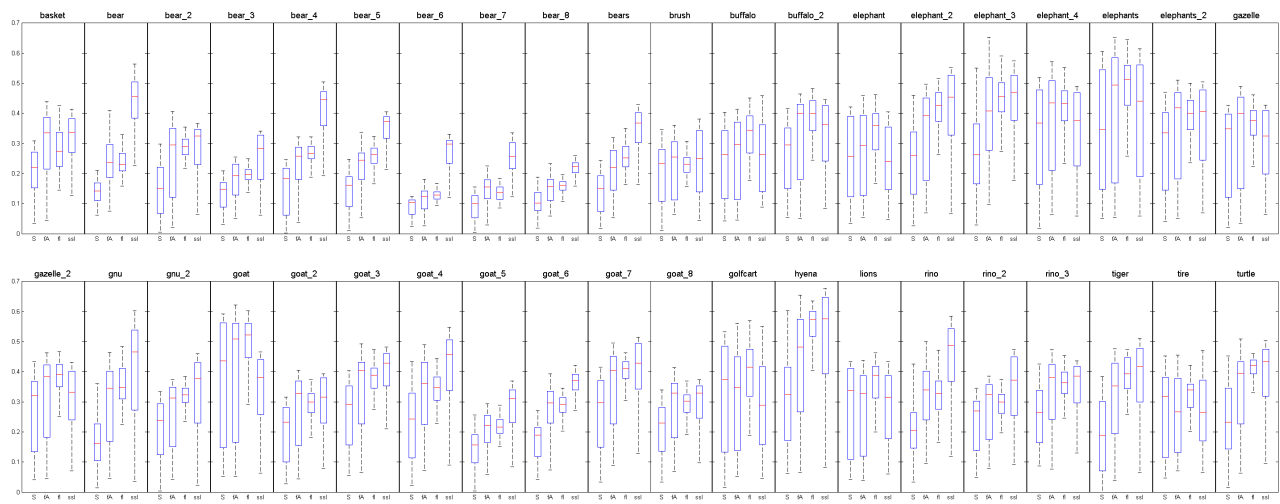

Figure 5: Contours comparison Left: from top to bottom, row 3 to 6 illustrate contours by $\mathbf{S}$ [四, proposed single-scale anisotropic fA, isotropic fI, and scale-space analysis ssI. Row 6 shows the best contours. Right: Box-and-whistler plot of all images. The proposed detectors outperform $\mathbf{S}$ in most cases, with ssI provides the most reliable contours. 


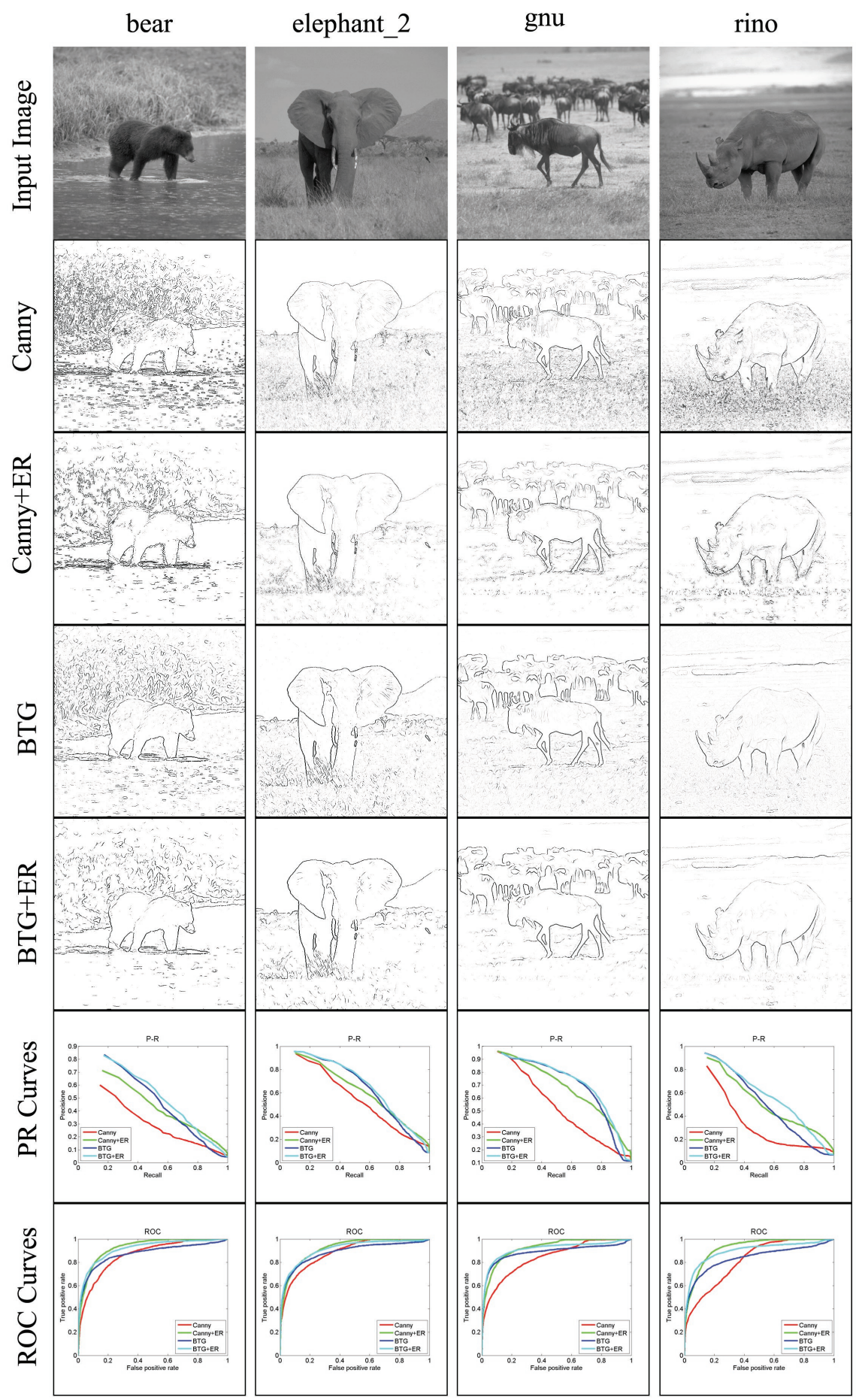

Figure 6: Improved contour detectors. Contours detected by early recurrent modulation (Canny+ER and BTG+ER) are significantly improved, indicating the proposed work defines a general approach to boost low-level feature extractions. 
bitions are two weighting schemes studied. They facilitate edge detection differently, with anisotropic provides better output for most test images. Additional experiments are required to further explore these schemes as well as other weighting methods.

Different from [四], early recurrent modulation boosts contour detection performance without any learning processing. To an extent, this study implies that even though the recent machine vision trend is in favor to learn low level features using large number of test samples, insights from the primate visual system still require further investigations and may be shown with great importance to the whole picture of visual interpretations.

\section{References}

[1] Grigorescu, C., Petkov, N., Westenberg, M.A.: Contour detection based on nonclassical receptive field inhibition. IEEE Trans. Image Process. 12 (2003) 729-739

[2] Papari, G., Campisi, P., Petkov, N., Neri, A.: A Biologically Motivated Multiresolution Approach to Contour Detection. EURASIP Journal on Advances in Signal Processing 07 (2007)

[3] Petkov, N., Subramanian, E.: Motion detection, noise reduction, texture suppression and contour enhancement by spatiotemporal Gabor filters with surround inhibition. Biological Cybernetics 97(5-6) (2007), 423-439

[4] Canny, J.: A computational approach to edge detection. IEEE Trans. Pattern Anal. Mach. Intell. 8 (1986) 679-698

[5] Mehrotra, R., Namuduri, K., Ranganathan, N.: Gabor filter-based edge detection. Pattern Recog. 25 (1992) 1479-1494

[6] Marr, D., Hildreth, E.: Theory of edge detection. Proc. R. Soc. Lond. [Biol.]. 207 (1980) pp. 187-217

[7] Konishi, S., Yuille, A., Coughlan, J., Zhu, S.C.: Statistical edge detection: learning and evaluating edge cues. IEEE Trans. Pattern Anal. Mach. Intell. 25 (2003) 57-74

[8] Thune, M., Olstad, B., Thune, N.: Edge detection in noisy data using finite mixture distribution analysis. Pattern Recog. 30 (1997) 685-699

[9] Ren, X.: Multi-scale improves boundary detection in natural images. In:Proc. ECCV '08, (2008) 533-545

[10] ArbelaİĄ andez, P., Maire, M., Fowlkes, C., Malik, J.: Contour detection and hierarchical image segmentation. IEEE Trans. Pattern Anal. Mach. Intell. 33 (2011) 898-916

[11] Ren, X., Fowlkes, C.C., Malik, J.: Learning probabilistic models for contour completion in natural images. Int. J. Comput. Vision 77 (2008) 47-63

[12] Martin, D., Fowlkes, C., Malik, J.: Learning to detect natural image boundaries using local brightness, color, and texture cues. IEEE Trans. Pattern Anal. Mach. Intell. 26 (2004) 530-549

[13] Ungerleider, L.G., Mishkin, M.: 18. In: Two Cortical Visual Systems. (1982) 549-586 
[14] Nowak, L., Munk, M., Girard, P., Bullier, J.: Visual latencies in areas v1 and v2 of the macaque monkey. Visual Neurosci. 12 (1995) 371-384

[15] Hupé, J.M., James, A.C., Payne, B.R., Lomber, S.G., Girard, P., Bullier, J.: Cortical feedback improves discrimination between figure and background by V1, V2 and V3 neurons. Nature 394 (1998) 784-787

[16] Bullier, J., HupÃl', J.M., James, A.C., Girard, P.: Chapter 13 the role of feedback connections in shaping the responses of visual cortical neurons. In C. Casanova, M.P., ed.: Vision: From Neurons to Cognition. Volume 134 of Progress in Brain Research. Elsevier (2001) 193-204

[17] Bullier, J.: Integrated model of visual processing. Brain Res. Rev. 36 (2001) 96-107

[18] Witkin, A.: Scale-space filtering: A new approach to multi-scale description. In: ICASSP'84. Volume 9. (1984) 150-153

[19] Zeng, C., Li, Y., Li, C.: CenterâĂŞsurround interaction with adaptive inhibition: A computational model for contour detection. NeuroImage 55 (2011) 49-66

[20] Essen, D.C.V., Maunsell, J.H.: Hierarchical organization and functional streams in the visual cortex. Trends Neurosci. 6 (1983) 370-375

[21] Felleman, D.J., Van Essen, D.C.: Distributed hierarchical processing in the primate cerebral cortex. Cerebral cortex (New York, N.Y. : 1991) 1 (1991) 1-47

[22] Albright, T.D.: Direction and orientation selectivity of neurons in visual area mt of the macaque. J. Neurophysiol. 52 (1984) 1106-1130

[23] Raiguel, S.E., Xiao, D.K., Marcar, V.L., Orban, G.A.: Response latency of macaque area $\mathrm{mt} / \mathrm{v} 5$ neurons and its relationship to stimulus parameters. J. Neurophysiol. 82 (1999) 1944-1956

[24] Kolster, H., Peeters, R., Orban, G.A.: The retinotopic organization of the human middle temporal area mt/v5 and its cortical neighbors. J. Neurosci. 30 (2010) 9801-9820

[25] Gabbiani, F., Krapp, H.G., Koch, C., Laurent, G.: Multiplicative computation in a visual neuron sensitive to looming. Nature 420 (2002) 320-324

[26] McAdams, C.J., Maunsell, J.H.R.: Effects of attention on orientation-tuning functions of single neurons in macaque cortical area v4. J. Neurosci. 19 (1999) 431-441

[27] Jones, J.P., Palmer, L.A.: An evaluation of the two-dimensional gabor filter model of simple receptive fields in cat striate cortex. J. Neurophysiol. 58 (1987) 1233-1258

[28] Valois, R.L.D., Albrecht, D.G., Thorell, L.G.: Spatial frequency selectivity of cells in macaque visual cortex. Vision Res. 22 (1982) 545-559

[29] Kaplan, E., Marcus, S., So, Y.T.: Effects of dark adaptation on spatial and temporal properties of receptive fields in cat lateral geniculate nucleus. J. Physiol. 294 (1979) $561-580$ 